\title{
Significantly enhanced sensor signal detection method using harmonic frequency in lock-in amplifier
}

\author{
Su-Jin Jeon, Jihoon Kim, Eudum Kim, Sun-Ho Kim, \\ Do-Hyun Kim, and Young-Wan Choi ${ }^{\text {a }}$
}

Lab. of Optoelectronics \& Circuit Systems (LOCS), Department of Electrical and Electronics Engineering, Chung-Ang University, 84 Heukseok-ro, Dongjack-gu, Seoul 06974, Korea

a)ychoi@cau.ac.kr

Abstract: In this study, we present a significantly enhanced signal detection method using harmonic frequency in a lock-in amplifier (LIA). This method reduces the effect of flicker noise that limits the signal-to-noise ratio in a conventional DC-based LIA. Furthermore, it has a definite advantage that it is not necessary to match the phase between the input and the reference signals. To verify such merits, we constructed a hybrid LIA circuit and conducted experiments. The results demonstrated that the dynamic range improved by approximately $43 \mathrm{~dB}$ compared to a conventional DC-based LIA. Moreover, the harmonic LIA output is successfully obtained regardless of the phase difference.

Keywords: $2 \omega_{\mathrm{c}}$ signal detection technique, lock-in amplifier, optical signal detection, SNR improving method

Classification: Optical systems

\section{References}

[1] S. M. Borisov and O. S. Wolfbeis: "Optical biosensors," Chem. Rev. 108 (2008) 423 (DOI: 10.1021/cr068105t).

[2] G. Krause, et al:: "1/f noise analysis of a $75 \mathrm{~nm}$ twin-flash ${ }^{\mathrm{TM}}$ technology nonvolatile memory cell," 7th Annu. Non-Volatile Mem. Technol. Symp. NVMTS (2006) 12 (DOI: 10.1109/NVMT.2006.378867).

[3] L. S. Levitov and G. B. Lesovik: "Charge distribution in quantum shot noise," Jetp Lett. C/C Pis'Ma V Zhurnal Eksp. Teor. Fiz. 58 (1993) 230.

[4] A. Van Der Ziel: "Thermal noise in field-effect transistors," Proc. IRE 50 (1962) 1808 (DOI: 10.1109/JRPROC.1962.288221).

[5] Stanford Research Systems: "About lock-in amplifiers," Appl. Note. (2011) 1.

[6] G. Giusi, et al:: "Ultra-low-noise large-bandwidth transimpedance amplifier," Int. J. Circuit Theory Appl. 43 (2015) 1455 (DOI: 10.1002/cta.2015).

[7] E. Kamrani, et al.: "Low-noise, high-gain transimpedance amplifier integrated with SiAPD for low-intensity near-infrared light detection," IEEE Sensors J. 14 (2014) 258 (DOI: 10.1109/JSEN.2013.2282624). 
(DOI: 10.1016/S0924-4247(01)00581-7).

[9] M. L. Meade: "Lock-in amplifiers: principles and applications," Peter Peregrinus Ltd on behalf of the Institution of Electrical Engineers.

[10] F. N. Hooge, et al.: "Experimental studies on 1/f noise," Rep. Prog. Phys. 44 (1981) 479 (DOI: 10.1088/0034-4885/44/5/001).

[11] H.-H. Son, et al.: "Signal detection technique utilising 'lock-in' architecture using $2 \omega_{\mathrm{c}}$ harmonic frequency for portable sensors," Electron. Lett. 46 (2010) 891 (DOI: 10.1049/el.2010.0684).

[12] P. C. D. Hobbs: "Photodiode front ends: The real story," Opt. Photonics News 12 (2001) 44 (DOI: 10.1364/OPN.12.4.000044).

[13] J. G. Graeme: Amplifier Applications of Op Amps (McGRAW-Hill, 1999).

[14] A. Bhat: "Stabilize your transimpedance amplifier," Maxim Integr. (2012).

[15] D. Wenn: "Implementing digital lock-in amplifiers using the dsPIC® DSC," AN115, Microchip (2007) 1.

[16] H.-K. Chiou and H.-Y. Chung: " $2.5-7 \mathrm{GHz}$ single balanced mixer with integrated Ruthroff-type balun in $0.18 \mu \mathrm{m}$ CMOS technology," Electron. Lett. 49 (2013) 474 (DOI: 10.1049/el.2012.4091).

[17] S.-G. Mao, et al.: "Design and modeling of uniplanar double-balanced mixer," IEEE Microw. Guided Wave Lett. 8 (1988) 354 (DOI: 10.1109/75.735419).

\section{Introduction}

Optical bio and chemical sensors possess several advantages, such as high sensitivity, small size, and fast response. Thus, they have recently received significant attention with regard to future applications in point-of-care (PoC) and Internet-of-Things (IoT) [1]. For high-sensitivity applications, optical sensor systems should detect exceedingly small or weak signals in a noisy environment. Various internal and external noises such as flicker noise, shot noise, and thermal noise, which limit the signal-to-noise ratio (SNR) of the system, are inevitably accompanied $[2,3,4]$. Therefore, a detection circuit system to reduce the effect of various noises is required. As a simple method, a modulation technique using a shunt resistor and bandpass filter (BPF) has been used for detecting the current signal generated by the optical sensor. However, this method has a certain limitation in case of detecting small or weak signals because it has no gain and a slow response time [5]. A trans-impedance amplifier (TIA) with a gain and fast response time is commonly used for current-to-voltage conversion instead of shunt resistor [6, 7]. Furthermore, it is well known that lock-in amplifier (LIA) certainly reduces the thermal noise effects [8]. The output signal of a conventional LIA is represented via a DC signal because the modulated signal passes through a phase sensitive detector (PSD) and a low-pass filter (LPF) [9]. Here, phase matching between the input and the reference signals are essential to maximize the output signal of the PSD. As another option, I/Q signal detections for vector summation have been applied so as not to use the phase matching circuits. Nevertheless, the SNR of the LIA is limited by the flicker noise, which is an intrinsic characteristic of semiconductor devices [10].

In this study, we present a signal detection method using harmonic frequency in a LIA. To avoid the flicker noise effect, which is inversely proportional to the 
frequency, we utilize a harmonic signal at $2 \omega_{\mathrm{c}}$ instead of DC signal [11]. Here, $\omega_{\mathrm{c}}$ is the modulation frequency of the input and the reference signal. This detection scheme distinctly indicates that the SNR limitation owing to the flicker noise can be overcome. Moreover, this method is not affected by the phase difference between the input and the reference signals. Therefore, the harmonic LIA would be a very efficient method because the phase controller for the phase matching between the input and the reference signals or dual channels for I/Q detection are not required. The harmonic LIA has been designed and fabricated as a hybrid circuit, and those advantages are successfully demonstrated in this study.

\section{Design of trans-impedance amplifier}

As the first step to construct an optical signal detection system, we design a TIA. In optical detection systems, TIA is commonly used at the front-end for current-tovoltage conversion. Fig. 1 shows a typical structure and transfer function diagram of a TIA using op-amp with feedback resistor and capacitor. Mathematically, the gain of the TIA is decided by the input impedance $\left(\mathrm{Z}_{\mathrm{I}}\right)$ and the feedback factor $(\beta)$ as given in equations (1) to (3). If the impedance of the feedback resistor $\left(\mathrm{R}_{\mathrm{F}}\right)$ is very large compared to the impedance of the input capacitance $\left(\mathrm{C}_{\mathrm{IN}}\right)$ and feedback capacitor $\left(\mathrm{C}_{\mathrm{F}}\right), \mathrm{R}_{\mathrm{F}}$ becomes a dominant factor deciding the gain of the TIA.

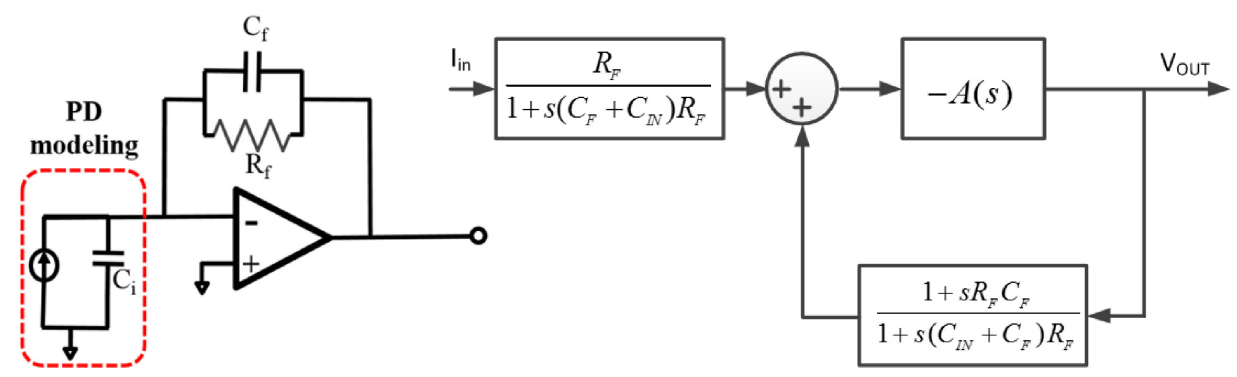

Fig. 1. Structure and transfer function diagram of TIA.

$$
\begin{gathered}
\text { Input impedance } Z_{\mathrm{I}}=\frac{R_{F}}{1+s\left(C_{F}+C_{I N}\right) R_{F}} \\
\text { Feedback factor } \beta=\frac{1+s R_{F} C_{F}}{1+s\left(C_{I N}+C_{F}\right) R_{F}} \\
\text { Gain of the TIA }(\mathrm{dB} \Omega) \frac{V_{\text {out }}}{I_{\text {in }}}=-Z_{I} \frac{A(s)}{1+\beta A(s)} \\
=\frac{R_{F}}{1+s\left(C_{F}+C_{I N}\right) R_{F}}\left[\frac{A(s)}{1+\frac{\left(1+s R_{F} C_{F}\right) A(s)}{1+s\left(C_{I N}+C_{F}\right) R_{F}}}\right]
\end{gathered}
$$

The bandwidth of the TIA is affected not only by $\mathrm{R}_{\mathrm{F}}, \mathrm{C}_{\mathrm{IN}}$, and $\mathrm{C}_{\mathrm{F}}$, but also by the gain bandwidth product (GBWP) of the op-amp. The bandwidth is widened according to the GBWP and feedback capacitor. Fig. 2 shows the frequency responses of gain and noise in the TIA. Noise gain is decided by $1 / \beta$. Here, $\mathrm{f}_{\mathrm{c}}$ is the cut off frequency of flicker noise and $\mathrm{f}_{\mathrm{z}}$ is the zero position of $1 / \beta$. Noise gain is 
minimized in the frequency region between $f_{c}$ and $f_{z}$ and increases in the other regions $[12,13,14]$. As shown in equations (4) to (6), the zero and pole positions of the TIA can be calculated using the feedback components and characteristics of the op-amp. Moreover, the SNR can be improved when the signal is detected and measured in the region of minimum noise gain.

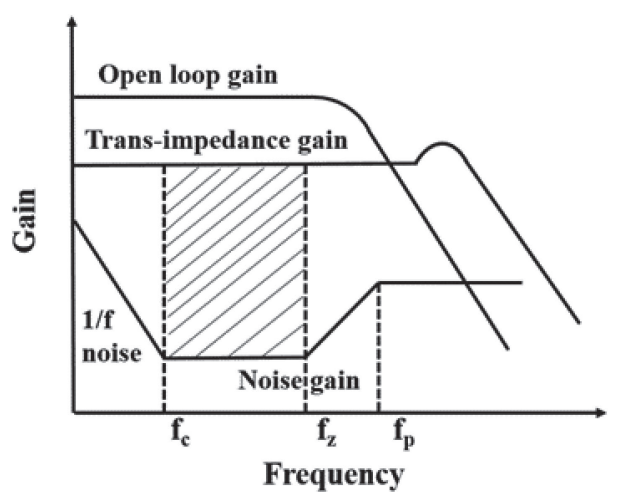

Fig. 2. Frequency response of gain and noise in TIA.

$$
\begin{gathered}
C_{F}=\frac{1}{4 \pi R_{F} f_{G B W}}+\sqrt{\frac{1}{\left(16 \pi R_{F} f_{G B W}\right.}+\frac{C_{I N}}{2 \pi R_{F} f_{G B W}}} \\
f_{p}=\frac{1}{2 \pi R_{F} C_{F}} \quad f_{z}=\frac{1}{2 \pi R_{F}\left(C_{F}+C_{I N}\right)} \\
f_{3 d B}=\frac{1}{2 \pi R_{F} C_{F}}=\frac{1}{2 \pi R_{F} \sqrt{\frac{C_{I N}}{2 \pi R_{F} f_{G B W}}}}=\sqrt{\frac{f_{G B W}}{2 \pi R_{F} C_{I N}}}
\end{gathered}
$$

As shown in Fig. 3, the TIA and amplifier are designed using op-amp LMH6629 and AD8034. A amplifier is employed at the end of the TIA for a signal gain. The optical sensor is modeled via the commonly used laser diode (LD) and photodetector (PD). The parasitic capacitance of the $\mathrm{PD}\left(\mathrm{C}_{\mathrm{in}}\right)$ is $10 \mathrm{pF}$, and the feedback components $\mathrm{R}_{\mathrm{f}}$ and $\mathrm{C}_{\mathrm{f}}$ are $4.7 \mathrm{k} \Omega$ and $1.2 \mathrm{pF}$, respectively. Fig. 4 shows the outputs of the TIA and the amplifier, respectively, for the optical input power of $-44 \mathrm{dBm}$ and the modulation frequency of $100 \mathrm{kHz}$. The TIA and buffer gain are $72 \mathrm{~dB} \Omega$ and $11 \mathrm{~dB}$, respectively.

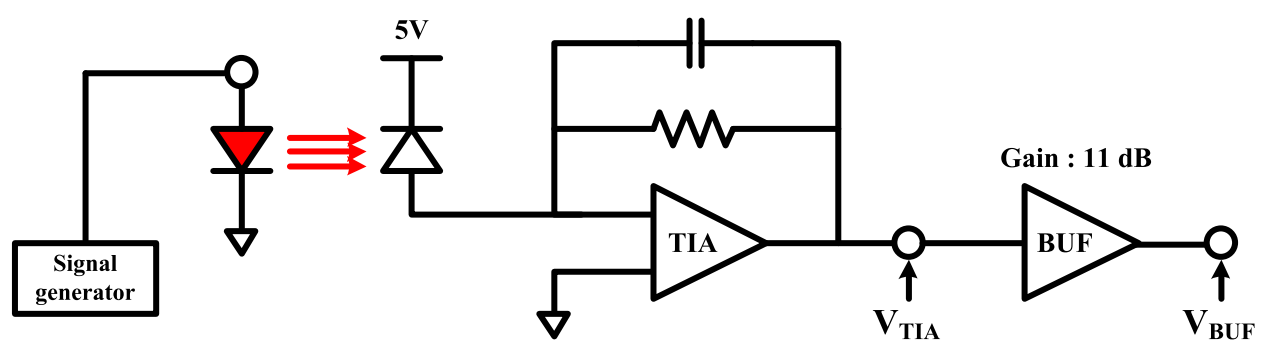

Fig. 3. Experimental setup of TIA with amplifier. 

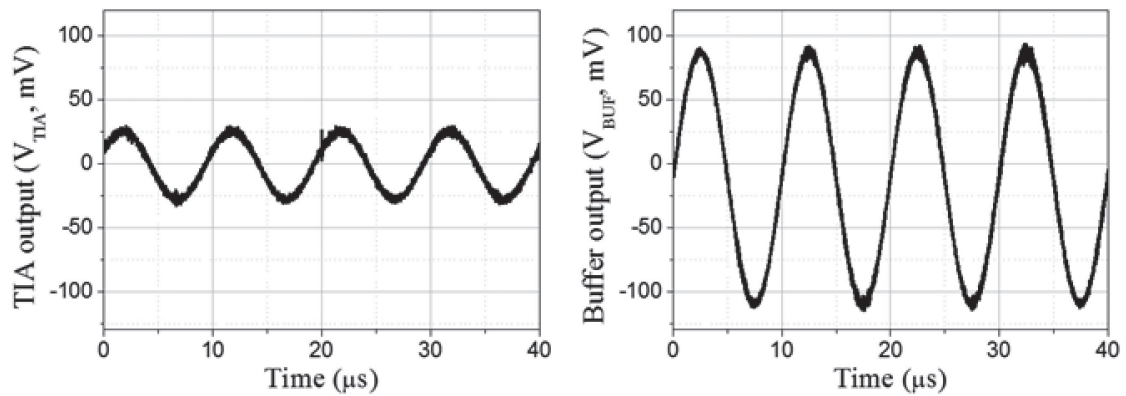

Fig. 4. Experimental results of TIA and amplifier output for optical input power of $-44 \mathrm{dBm}$ and modulation frequency of $100 \mathrm{kHz}$.

\section{Design of lock-in amplifier and detection method using harmonic frequency signal}

Fig. 5 shows the block diagram of a LIA, which is widely used for detecting a weak signal hidden in various noise. The measurement method requires having the same frequency for the input and the reference signals. The input signal is amplified and multiplied with the lock-in reference signal using the PSD. For the input and reference signals in equation (7), the PSD output is given by equation (8). The output signal of the PSD has both the difference and sum frequencies of the input and the reference signals.

$$
\begin{gathered}
V_{S I G}=A_{S I G} \cos \left(\omega_{\mathrm{c}} t+\theta_{S I G}\right), \quad \mathrm{V}_{R E F}=A_{R E F} \cos \left(\omega_{\mathrm{c}} t+\theta_{R E F}\right) \\
V_{P S D}=A_{S I G} \cos \left(\omega_{\mathrm{c}} t+\theta_{S I G}\right) \times A_{R E F} \cos \left(\omega_{\mathrm{c}} t+\theta_{R E F}\right) \\
=\frac{1}{2} A_{S I G} A_{R E F}\left[\cos \left(\theta_{S I G}-\theta_{R E F}\right)+\cos \left(2 \omega_{\mathrm{c}} t+\theta_{S I G}+\theta_{R E F}\right)\right] \\
V_{P S D}=\frac{1}{2} A_{S I G} A_{R E F}+\frac{1}{2} A_{S I G} A_{R E F} \cos \left(2 \omega_{\mathrm{c}} t+2 \theta_{S I G}\right), \quad\left(\text { if } \theta_{S I G}=\theta_{R E F}\right)
\end{gathered}
$$

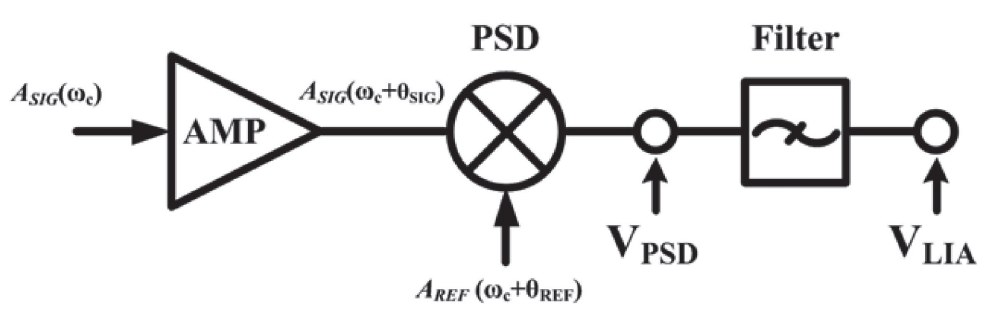

Fig. 5. Block diagram of lock-in amplifier.

If the phase difference between the input and reference signal is zero, the PSD output can be expressed as in equation (9) with a maximum DC value and a component at $2 \omega_{\mathrm{c}}$ frequency. Here, $\omega_{\mathrm{c}}$ is the frequency of the input and reference signal. Therefore, we can detect a weak signal by obtaining the DC signal through the LPF under the condition of phase matching. If the phase difference is not zero, the DC output signal of the PSD varies in accordance with the phase difference as shown in equation (8). It implies that a phase controller is essential in a single channel LIA [15]. For a dual channel LIA, where the vector sum of the I and Q channels is available, additional $90^{\circ}$ out-of-phase controller and PSD are required. Evidently, it makes the system more complex. On the other hand, if we utilize the 


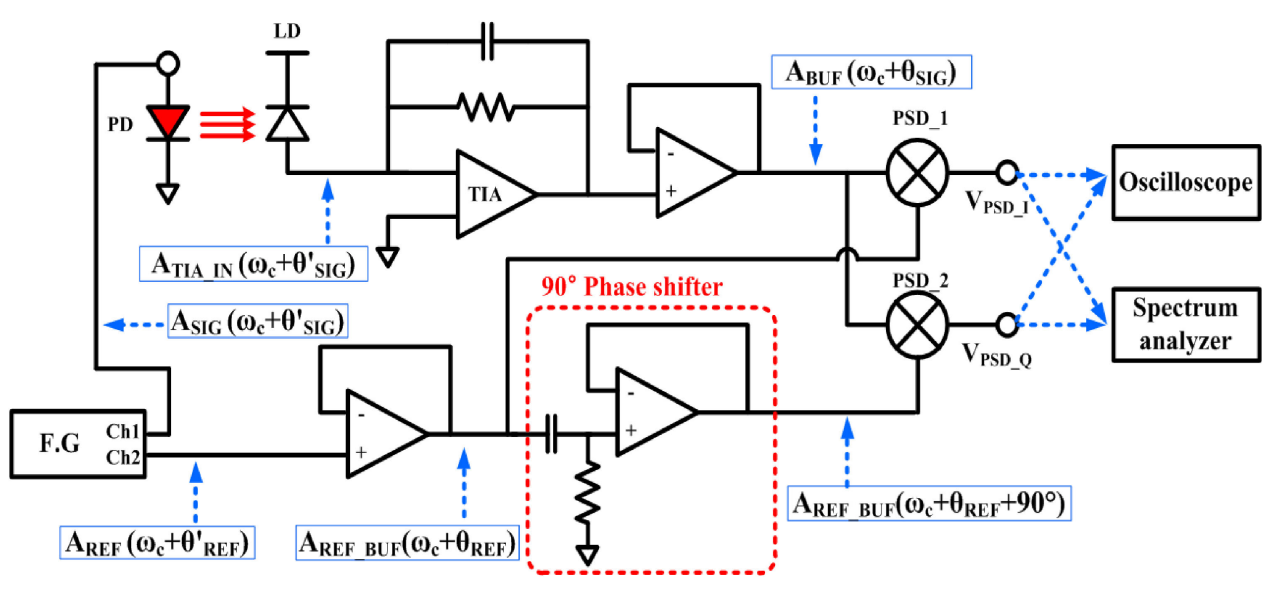

Fig. 6. Experimental setup of dual channel LIA. $V_{P_{P S} D_{-}}$in lower path has phase difference of $90^{\circ}$ with $\mathrm{V}_{\text {PSD_I }}$.

harmonic frequency $\left(2 \omega_{\mathrm{c}}\right)$ component, the SNR can be significantly improved. Because it is free from the flicker noise which is inversely proportional to the frequency and exists only at low frequency regions.

To compare the signal of each LIA and to demonstrate the advantages of the harmonic LIA, we construct a circuit as shown in Fig. 6. The figure illustrates the schematic of the dual channel LIA. Also, it includes phase adjustment circuit for the $90^{\circ}$ phase difference between the reference signals of I/Q channels. The PSD used in the experiment is constructed using the analog multiplier AD633. An output of the analog multiplier has no harmonics other than $2 \omega_{\mathrm{c}}$. Here, it should be noted that several other harmonic signals can appear if the PSD is based on a switch mixer such as single and double balanced mixer [16, 17]. These switch mixers are exceptionally sensitive to the phase matching thereby hindering the advantage of harmonic signal detection in LIA. Moreover, an additional BPF is required for the selection of $2 \omega_{\mathrm{c}}$ harmonic frequency. This is the reason for utilizing multiplier instead of switch mixer. The experimental conditions are as follows: the input optical power is $-50 \mathrm{dBm}$, the modulation frequency is $100 \mathrm{kHz}$, and the reference signal amplitude is $200 \mathrm{mV}_{\mathrm{pp}}$.

The entire circuit as shown in Fig. 6 is required for the vector sum of I/Q signals in a dual channel LIA. For a single channel operation of the LIA, VPSD_I output and a phase controller for the same phase of the PSD inputs $\left(\theta^{\prime}{ }_{\text {SIG }}=\theta^{\prime}{ }_{\mathrm{REF}}\right)$ are required instead of the buffer and $90^{\circ}$ phase shifter in the lower path of the circuit in Fig. 6. On the other hand, if we use a harmonic frequency, only the upper path output signal $\mathrm{V}_{\text {PSD_I }}$ is required because the harmonic signal can be obtained regardless of the phase change. The phase change does not affect the peak-to-peak amplitude of the harmonic signal. Therefore, circuits for the phase matching are not required as long as the amplitude of the harmonic signal can be measured.

\section{Experimental results}

Fig. 7 shows the PSD outputs measured using a spectrum analyzer according to the variation of the optical input power. The phases of laser modulation and reference signals were set to be equal $\left(\theta^{\prime}{ }_{\text {SIG }}=\theta^{\prime}{ }_{\mathrm{REF}}\right)$. The magnitude of flicker noise in our 


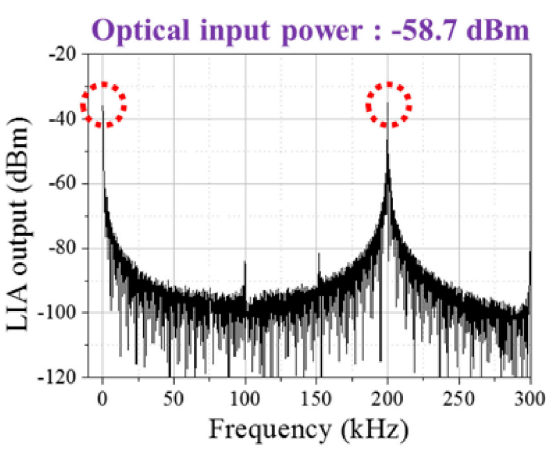

(a)

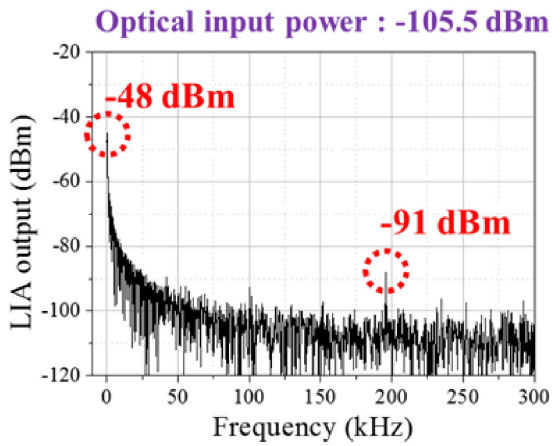

(c)

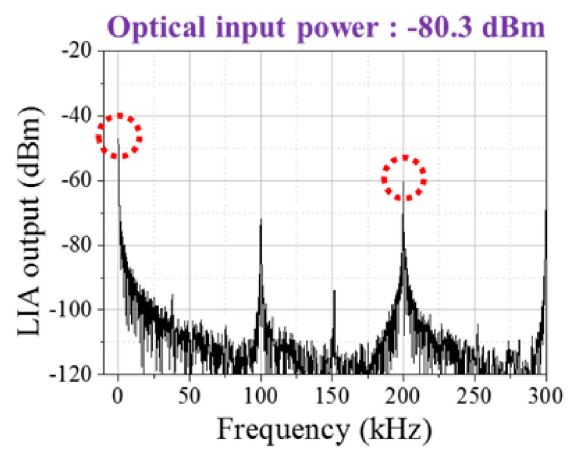

(b)

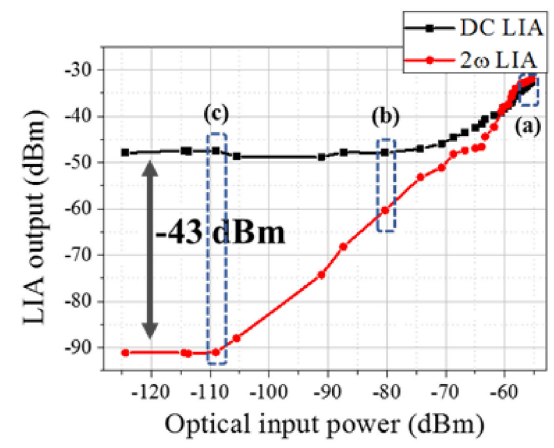

(d)

Fig. 7. Experimental results of DC and $2 \omega_{\mathrm{c}}$ LIA with spectrum analyzer according to optical input power. (a) $-58.7 \mathrm{dBm}$ (b) $-80.3 \mathrm{dBm}$ (c) $-105.5 \mathrm{dBm}$. (d) Experimental results of DC and $2 \omega_{\mathrm{c}}$ LIA output versus optical input power.

measurement system was approximately $-48 \mathrm{dBm}$ at DC. It is well known that flicker noise varies depending on the circuit, instrumentation, and experimental environment. Fig. 7 distinctly indicates that the output power at DC decreases as the optical input power decreases until the flicker noise limitation is attained. Therefore, the detection limit was determined via the flicker noise. On the other hand, the output power at the harmonic frequency of $200 \mathrm{kHz}\left(2 \omega_{\mathrm{c}}\right)$ is measured as low as $-91 \mathrm{dBm}$. The dynamic range is significantly improved by approximately $43 \mathrm{~dB}$. The magnitude of about $-48 \mathrm{dBm}$ at Dc is only about $0.9 \mathrm{mV}$. Moreover, the detected signal $-91 \mathrm{dBm}$ at $2 \omega_{\mathrm{c}}$ is only about $6.3 \mu \mathrm{V}$. It is evident that no effect of the flicker noise at the $2 \omega_{\mathrm{c}}$ harmonic frequency exists.

Fig. 8 shows the output of the DC LIA and $2 \omega_{\mathrm{c}}$ LIA according to the phase difference between the input signal and the reference signal $\left(\theta_{\text {SIG }}=0^{\circ}, \theta_{\text {REF }}=\right.$ variation). As the phase changes, it is experimentally confirmed that the peak-topeak amplitude of the PSD outputs ( $\mathrm{V}_{\text {PSD_I }}, \mathrm{V}_{\mathrm{PSD}} \mathrm{Q}$ ) are not changed, while the DC level is changed. Regarding the DC signal, the amplitude of each channel modulates with the phase difference. When the phase difference is set to zero, the DC values of I/Q channels are $8.9 \mathrm{mV}$ and $-1.0 \mathrm{mV}$, respectively. As expected, these values are changed to $-0.1 \mathrm{mV}$ and $8.8 \mathrm{mV}$, respectively, as the phase difference becomes $90^{\circ}$. However, the vector sum obtained via the I/Q dual channels remains almost the same as long as the phases of the reference signal in the I/Q channels is $90^{\circ}$ apart. Though the theoretical value of the vector sum is approximately $10.7 \mathrm{mV}\left(\mathrm{V}_{\mathrm{pp}} / 2\right)$, the average value of the vector sum is approximately $9 \mathrm{mV}$. The parasitic components generated in the circuits and a slight phase 

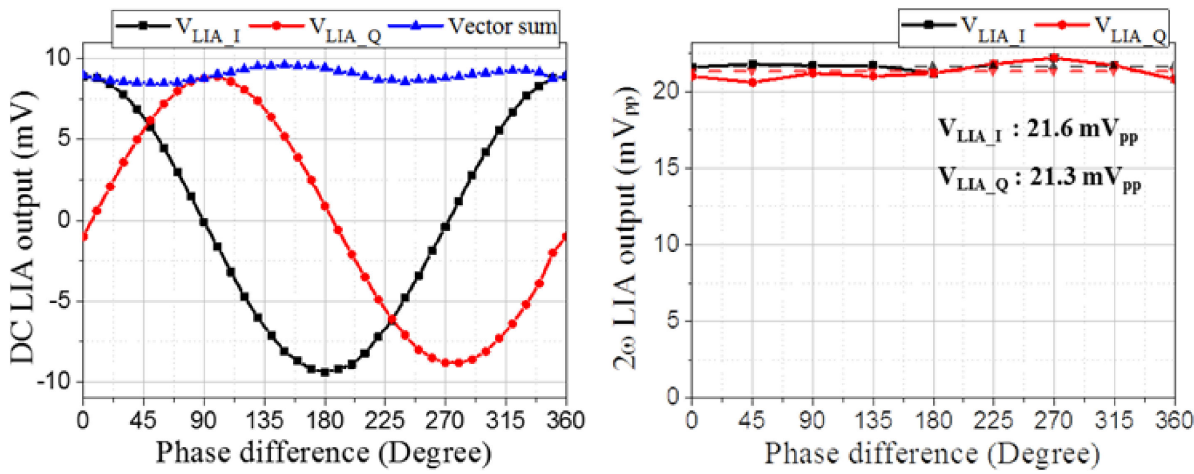

Fig. 8. Experimental results of vector sum in DC LIA and $2 \omega_{\mathrm{c}}$ LIA according to phase difference between input signal and reference signal.

mismatching in the $90^{\circ}$ phase shifter are considered as the cause of the error. Regarding the $2 \omega_{\mathrm{c}}$ harmonic frequency signal, the peak-to-peak amplitude does not vary with the phase difference and the average values of the I/Q channels were approximately $21.6 \mathrm{mV}_{\mathrm{pp}}$ and $21.3 \mathrm{mV}_{\mathrm{pp}}$, respectively. Therefore, it is not necessary to use the dual channel because the peak-to-peak amplitudes of $2 \omega_{\mathrm{c}}$ signals are the same regardless of the phase differences. Note that these results are obtained using multiplier mixer instead of switch mixer. Our experiment distinctly indicates the definite advantage of the harmonic detection method in LIA.

\section{Conclusions}

In conclusion, we demonstrated the signal detection method using harmonic frequency to reduce the effect of the flicker noise in the LIA. This method is not affected by phase difference between the input and reference signals, and significantly enhances the detection limit owing to the flicker noise. It was distinctly experimentally verified that the dynamic range was improved by approximately $43 \mathrm{~dB}$ compared to the DC-based conventional LIA. Moreover, the requirement of dual channels for vector summation was eliminated. These results imply that the sensitivity of an optical sensor detection system can be improved, and the system size can be reduced. Therefore, the signal detection method using harmonic frequency would be very useful for portable optical sensor system.

\section{Acknowledgments}

This research was supported by the Korea Institute for Advancement of Technology (KIAT) grant funded by the Korean government (MOTIE: Ministry of Trade, Industry \& Energy, HRD Program for Software-SoC convergence) (No. N0001883) and by Basic Science Research Program through the National Research Foundation of Korea (NRF) funded by the Ministry of Education (NRF2018R1D1A1B07048145) and by Chung-Ang University Graduate Research Scholarship in 2017. 